\title{
COME DOVEVA COMPORTARSI UNA DONNA E QUALI ERANO I SUOI DOVERI? INDICAZIONI SUGGERITE AL PUBBLICO FEMMINILE IN ALCUNE PREDICHE DI GILBERTO DI TOURNAI ${ }^{1}$
}

Lo scopo di questo articolo è di presentare quali erano i modi di comportamento richiesti alle donne per essere considerate ben educate e quali erano i loro doveri secondo il pensiero di Gilberto di Tournai († I284), uno dei più famosi predicatori francesi del XIII secolo. Il suo insegnamento è uno dei rari testi medievali pervenuti ai nostri tempi che ci permette di conoscere quale comportamento delle donne era ritenuto giusto e invece quale sbagliato dalla società del tempo come pure quali doveri avevano le ragazze, le spose e le vedove.

A causa della scarsità delle testimonianze scritte dalle donne vissute in epoca medievale che getterebbero più luce sulla questione che interessa il presente studio, è necessario esaminare i testi degli uomini che nelle loro opere hanno trattato questo argomento. Infatti, dal XIII secolo gli autori e i predicatori notarono la mancanza dei testi educativi e cominciarono a scrivere più spesso opere di contenuto pedagogico, rivolgendosi però all'inizio soprattutto ai giovani provenienti dalle classi sociali più alte. In questo periodo infatti, composero le loro opere ad esempio Vincent de Beauvais il quale scrisse De eruditione filiorum nobilium, o Egidio Romano, l'autore del De regimine principum, o Paolo da Certaldo che pubblicò il Libro di buoni costumi. A causa della scarsità delle opere di questo genere anche alcuni genitori decisero di scrivere i loro suggerimenti ai propri figli e così, ad esempio, nel XIII secolo Bellini Bisolo scrisse il Liber legum moralium. Similmente accadde riguardo alle opere dedicate al pubblico femminile. Infatti, dal XIII secolo gli scrittori si rivolsero alle donne più frequentemente cercando di dar loro consigli soprattutto circa i costumi, le buone maniere, la formazione religiosa e morale ${ }^{2}$. Il testo più conosciuto dedicato esplicitamente alle donne è quello composto nel I320 dal fiorentino Francesco da Barberino intitolato Regimento e costumi di donna. Un'altra opera che ebbe una certa fama in quell'epoca è stato il Livre pour l'enseignement de ses filles di Geoffroy de La Tour Landry, composta per le figlie in età di matrimonio e il Libre de les dones di Francesco Eiximensis. Inoltre si conoscevano anche le opere indirizzate soltanto a una delle donne come ad esempio il testo di Menagier

L. Sileo, Maestri francescani, secolari e agostiniani, in: Storia della teologia nel medioevo, vol. 3: La teologia della scuole, cur. G. D’Onofrio, Casale Monferrato 1996, pp. 11-12; A. Horowski, Opere e manoscritti di Gilberto di Tournai: nota bibliografica integrativa, Roma 2015.

2 Per approffondire si invita di leggere E. Power, Donne del Medioevo, M.M. Postan cur., Milano 1999, pp. 71-84. 
de Paris, scritto per una moglie proveniente dalla borghesia ${ }^{3}$, la lettera di Anna di Francia, composta per la figlia Susanna ${ }^{4}$, la lettera di Luigi IX, destinata alla figlia Isabela ${ }^{5}$, il Livre des trois vertus composto da Christine de Pizan per la principessa Margherita di Borgogna o le lettere di Francesco Dattini alla moglie Margherita, scritte tra il I385 e il I4IO. Il bisogno di questo tipo di opere di contenuto educativo indirizzato alle donne era così ampio che Francesco da Barberino, nel Proemio di Reggimento si lamentò riguardo alla loro scarsità:
"Novellamente, Franciesco, parlai
Coll'Onestade,
ed a preghiera di molte altre donne
mi lamentai collei,
e dissi ch'erano molti
ch'aveano scritt' i' libri,
costumi ornati d'omo, ma non di donna".

Esaminando i testi medievali pervenuti ai nostri tempi si nota che nella maggior parte dei casi essi erano scritti o da ecclesiastici o da uomini che appartenevano alle classi sociali più elevate. Invero in quell'epoca l'educazione era prevista generalmente o per le persone che dovevano abbracciare la vita religiosa o per quelle provenienti da casati molto ricchi. Non sorprende allora che l'opinione riguardante le donne provenisse dalle due fonti principali: dall'aristocrazia e dalla chiesa. Invero l'aristocrazia, essendo la classe sociale più elevata e ricca, aveva una maggiore possibilità di ricevere una buona istruzione, lusso che soltanto pochi potevano permettersi. Infatti, assumere un insegnante o mandare i figli in un monastero per farli studiare richiedeva dal padre una spesa cospicua e perciò non tutti ritenevano cosa strettamente necessaria quella di far studiare ai figli, destinati a condurre vita mondana, le materie di trivium e quadrivium. Invero frequentemente i padri presero su se stessi il dovere dell'educazione dei figli, insegnando loro tutto il necessario che un uomo della loro classe sociale doveva sapere, lasciando invece il compito di istruire le figlie alle loro madri. Quando una famiglia decideva però di assumere un insegnante, spesso si rivolgeva ai monaci o ai preti disposti a vivere e rimanere nella loro casa per qualche anno istruendo i giovani. Questi tutori frequentemente davano ai loro studenti come esercizio di lettura testi misogini. Non stupisce allora che i ragazzi nutriti di queste idee crescessero guardando le donne con sospetto e le considerassero utili solo per la procreazione dei figli e per la guida della casa. Da parte loro i chierici, di solito celibi, non conoscevano e non capivano le donne poiché portati spesso nei conventi in tenera età, raramente rimanevano in contatto con loro. Infatti, che cosa poteva ricordarsi della sua madre o delle sue sorelle un uomo che sin dall'età infantile non le aveva più viste? Chiusi in un monastero, ascoltando in continuazione che l'uomo è stato cacciato dal paradiso per colpa di Eva, gli uomini vedevano la propria figlia in ogni donna. Inoltre la lettura dei testi dei Padri rafforzava sempre di più la loro ostilità contro il

\footnotetext{
3 Cfr. Le Ménagier de Paris, The good wife's guide, G. L. Greco, Ch. M. Rose translated with Critical Introduction, Cornell University 2009.

4 Cfr. Xodo Cegolon C., Lo specchio di Margherita, per una storia dell'educazione femminile nel Basso Medioevo, C.L.E.U.P., Padova 1988, pp. 87-125.

5 Cfr. Ibidem, pp. 126-129.

6 Francesco da Barberino, Reggimento e costumi di donne, C. Baudi di Vesme, G. Romagnoli (curr.), Bologna 1875, p. 3.
} 
sesso femminile. Non stupisce perciò che crescendo in quell'ambiente considerassero tutte le donne come la causa di ogni male che colpiva l'uomo e, nei loro testi, nelle loro prediche e perfino nei loro insegnamenti si nota spesso un pensiero misogino ${ }^{7}$.

Il presente studio riguarda il pensiero di uno dei più conosciuti predicatori vissuti nella Francia del XIII secolo, Gilberto di Tournai, il quale notò la mancanza di opere scritte per le donne e la trascuratezza della loro educazione da parte dei genitori e dei mariti. Seguendo il suo insegnamento, il presente studio sarà diviso in tre parti e tratterà delle ragazze in età di matrimonio, delle donne maritate e infine delle vedove. Questa divisione del pubblico femminile era applicata anche dagli altri predicatori e dagli scrittori attivi in epoca medievale, i quali con i loro testi si rivolgevano alle donne, come, ad esempio, Alano di Lilla $(\dagger \mathrm{I} 2 \mathrm{O} 2)^{8}$, Bernardino da Siena (†I444) ${ }^{9}$, frate Cherubino (†I484) ${ }^{\mathrm{IO}}$ o Giacomo di Vitry $(\dagger \mathrm{I} 240)^{\mathrm{II}}$. Altri scrittori dedicavano i loro testi seguendo lo status sociale dal quale esse provenivano e così ad esempio Umberto da Romans (†I277) nelle sue prediche destinate al pubblico femminile parlava alle figlie dei nobili, alle discendenti dei ricchi borghesi, alle loro domestiche, alle donne povere provenienti da piccoli villaggi, alle meretrici e alle monache $^{\mathrm{I} 2}$. Francesco da Barberino (†1348) invece nel Reggimento e costumi di donna era più dettagliato e si rivolgeva alle figlie di un re, di un barone, di un borghese, di un cavaliere, di un mercante, etc.

La scarsità di testi medievali composti per il pubblico femminile mostra che ancora pochi uomini ritenevano necessaria l'educazione delle donne alla lettura. Inoltre si nota che il loro pensiero al riguardo non era omogeneo e così alcuni di loro, come ad esempio Vincent de Beauvais, ritenevano utile insegnare loro a leggere e a scrivere, altri, come ad esempio Paolo da Certaldo, sostenevano che questo non era necessario per loro, mentre altri ancora, come Francesco da Barberino, cercavano una via di mezzo, permettendo alle donne provenienti da famiglie di alto status sociale d'imparare a leggere.

Le opere dedicate alle donne, per le quali era prevista la vita nel mondo, trattavano soprattutto della questione delle buone maniere e dei costumi. Infatti, gli autori e i predicatori descrivevano quali virtù e quali doveri dovevano avere le ragazze, le mogli e le vedove per essere considerate ben educate. Invero, anche se le aspettative dipendevano dallo status sociale della donna si possono notare alcune virtù, come la modestia e l'umiltà ${ }^{13}$, che dovevano essere presenti in tutte loro.

\footnotetext{
Per saperne di più s’invita a leggere: E. Power, Donne del medioevo, M.M. Postan (cur.), Milano19994; G. Duby, M. Perrot, Storia delle donne in Occidente. Il Medioevo Ch. Klapisch-Zuber (cur.), Roma-Bari 2005.

8 Cfr. Alano di Lilla, Summa de arte praedicatoria, in PL 210, 111-195.

9 Cfr. Bernardino da Siena, Le prediche volgari vol. 3, Firenze 1940; idem, Le prediche volgari vol. 2, Firenze 1958; idem, Prediche volgari sul campo di Siena 1427, a cura di C. Delcorno, vol. 2, Milano 1989.

10 Cfr. Regole della vita matrimoniale di Frate Cherubino da Siena, F. Zambrini, C. Negroni (curr.), Bologna 1888 .

11 Cfr. Jacobi de Vitriaco Sermones vulgares vel ad status, in CCCM Tournhout 2013.

12 Cfr. Umberto da Romans, in Prediche alle donne del secolo XIII. Testi di Umberto da Romans, Gilberto da Tournai, Stefano di Borbone, C. Casagrande (cur.), Milano 1978, p. 83.

13 Cfr. Gilberto da Tournai, Terza predica alle donne sposate, in Prediche alle donne del secolo XIII. Testi di Umberto da Romans, Gilberto da Tournai, Stefano di Borbone, C. Casagrande (cur.), Milano 1978, p. 85.
} 


\section{La ragazza}

Rivolgendosi alla ragazza in età di matrimonio Gilberto di Tournai poneva l'accento sulla sua verginità insistendo che la conservasse, non perché la fanciulla fosse costretta da qualcuno a farlo, ma perché l'aveva deciso lei stessa ${ }^{14}$. Dalla vergine inoltre si aspettavano le seguenti virtù: "una volontaria benevolenza, una vereconda semplicità, una misericordiosa carità, un'umile obbedienza, un'ilare pazienza, una bella coscienza, una previdente perseveran-

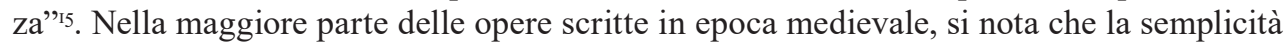
e l'umiltà erano le due virtù più ricercate nelle donne dagli uomini ${ }^{16}$. Non stupisce allora che alle ragazze spesso si desse l'esempio della paziente Giseldis, che con l'umiltà e la semplicità accettava la sua difficile sorte ${ }^{17}$. In seguito Gilberto rilevava che una ragazza ben educata non avrebbe dovuto essere rimproverata da nessuno per il suo modo di parlare, per i suoi gesti o per il suo modo di camminare ${ }^{\mathrm{I}}$. Inoltre essa doveva essere "pudica, ornarsi in modo che ogni cosa fosse in lei onesta e composta"19. L'opinione di Gilberto al riguardo non era diversa da quella degli altri predicatori e scrittori. Infatti, un famoso predicatore italiano, Bernardino da Siena, vissuto più di cento anni dopo, parlando del vestito delle ragazze insisteva sulla sua modestia, ma aggiungeva che il vestito doveva essere sempre adeguato allo status sociale della ragazza $^{20} \mathrm{e}$ doveva mostrare l'onestà della giovane donna ${ }^{21}$. Anche Anna di Francia trattava di pudore e suggeriva a sua figlia, Susanna, di non portare vestiti che "recano oltraggio, né troppo stretti né troppo cadenti" 22 perché la vera eleganza risiede nel "bel portamento, altero e onorevole, nei modi dolci, molto controllati e decisi in ogni cosa"²3. Umberto da Romans

14 Cfr. Ibidem, p. 83.

15 Ibidem.

16 Per vedere di più s'invita a leggere Egidio Romano, Del reggimento de' principi, F. Corazzini (cur.), Firenze 1858; Vincent of Beauvais, De eruditione filiorum nobilium, A. Steiner (ed.), Cambridge, Massachusets 1938, pp. 190-194 Francesco da Barberino, Reggimento e costumi di donne, a cura di C. Baudi di Vesme, G. Romagnoli, Bologna 1875; Ludovico Dolce, Dialogo della institution delle donne, in Vinegia 1547.

17 Secondo il racconto, il giovane marchese di Salise di nome Wuistasse, che non si voleva sposare, un giorno incontrò Griseldis, la figlia di un uomo onesto. La ragazza aveva quindici anni, era molto bella, di buoni costumi e si occupava con umiltà e devozione del padre e della casa sua. Il Marchese, colpito dalla sua bellezza e dalle sue virtù, la sposò. Presto nacque la loro prima figlia, ma il marchese decise di mettere Griseldis in prova per assicurarsi della sua obbedienza e umiltà. Un giorno disse alla consorte che non si poteva accettare che la sua discendente provenisse da origini così umili e lui per questo aveva deciso di uccidere la figlia. La moglie addolorata accettò la decisione del marito. Poco dopo la coppia ebbe un figlio e il marchese mise di nuovo la moglie alla stessa prova. Lei accettò. Ma lui non essendo ancora sicuro decise di metterla in un'altra prova e le disse di tornare a casa di suo padre. Griseldis obbedì. Il marchese ancora non convinto dell'umiltà ed obbedienza della moglie la informò che si voleva risposare con una giovane donna che era venuta accompagnata da suo fratello. Il Marchese fece venire al castello Griseldis e le ordinò di preparargli la festa del matrimonio insieme alle serve che lavoravano nella sua casa. Durante il pranzo nuziale il marchese chiese a Griseldis la sua opinione riguardo alla sua nuova moglie. La sua risposta commosse tutti, marchese compreso, perché la donna gli chiese di avere pietà della nuova consorte e di risparmiarle il dolore e la sofferenza che lei stessa dovette sopportare durante tutto il loro matrimonio. Solo in quel momento il marchese disse che era ormai sicuro di avere una moglie buona e che non voleva averne nessun'altra. Inoltre spiegò a tutti i presenti che la ragazza seduta accanto era veramente la loro figlia. Cfr. C. Xodo Cegolon, Lo specchio di Margherita, op.cit., p. 47-48.

18 Cfr. Gilberto da Tournai, Terza predica alle donne sposate, op.cit., p. 83.

19 Ibidem, p. 83.

20 Cfr. Bernardino da Siena, Prediche volgari, op.cit., p. 879.

21 Cfr. Ibidem, p. 887.

22 Anna di Francia, Insegnamenti alla figlia Susanna, op. cit., p. 95.

23 Ibidem, p. 95. 
invece da parte sua avvertiva che le donne che si occupavano troppo dei loro abbigliamenti dovevano temere di essere respinte dal regno dei cieli $^{24}$.

Un grave problema che doveva affrontare Gilberto, e con lui anche gli altri predicatori e scrittori medievali, era la curiosità delle ragazze e la loro voglia di uscire di casa per vagabondare per le vie e le piazze. Infatti, il predicatore francese rivolgendosi alle ragazze le avvertiva, ricordando la storia di Dina, figlia di Giacobbe, che incuriosita uscì con la sua famiglia di casa e mentre camminava, la notò il figlio di un re il quale s'innamorò di lei e la violentò ${ }^{25}$. Secondo Gilberto la ragazza e la sua famiglia dovevano ricordarsi sempre di questa storia e considerare ogni uscita di casa un vero pericolo. Infatti, come insisteva Gilberto, anche andando in chiesa per la santa messa, la giovane poteva essere vista da qualche uomo e suscitare in lui il desiderio carnale. La stessa opinione condivideva Bernardino da Siena, il quale consigliava ai genitori di custodire le loro figlie dentro le case e di non permettere loro neanche di avvicinarsi alle finestre o alle porte. Infatti, secondo lui la più bella ragazza era quella che non si faceva vedere da nessuno. Inoltre il predicatore avvertiva i genitori che mentre questi ascoltavano le sue prediche, le loro figlie a casa passavano il tempo vicino alle porte o alle finestre per essere viste dai giovani i quali le attiravano con i loro canti. La stessa opinione condivideva Egidio Romano, che da parte sua aggiungeva "l'uomo non die lassare andare le femmine molto correre, né andare attorno per la contrada né per le rughe"26. Anche Francesco da Barberino ironizzava riguardo alle donne che passavano il loro tempo vicino alle finestre perché "chi a fenestra cuce, spesse fiate si cuce la mano quand'ella crede suo vestito cucire" di uscire di casa era comune e sia i predicatori che gli scrittori del tempo con continuità lottavano contro questo "vizio" femminile.

Un'altra questione con la quale si doveva lottare secondo Gilberto riguardava i canti secolari. Questa però, come si vedrà in seguito, è una questione nella quale mancava l'omogeneità del pensiero dei predicatori e degli scrittori del tempo. Infatti, da parte sua il predicatore francese non permetteva alle ragazze di sentire e di cantare i canti "del secolo"28. Condivideva pienamente la sua opinione Giacomo di Vitry, il quale sosteneva con fermezza che "la donna che dà inizio al canto è la cappellana del diavolo e quelli che le rispondono i suoi sacerdoti" ${ }^{29}$. Francesco da Barberino però, da parte sua, riteneva che la ragazza dovesse esercitarsi nel "canto camerale" e le concedeva il permesso di ascoltare la musica. Inoltre lo scrittore fiorentino incoraggiava le ragazze ad imparare a suonare "mezzo cannone, la viola o l'altro istrumento onesto e bello"30.

Gilberto, come la maggiore parte degli uomini, riteneva che ogni donna cercasse d'ingannare gli uomini. Secondo lui la ragazza esercitandosi davanti allo specchio per cercare

\footnotetext{
24 Cfr. Umberto da Romans, Alle fanciulle o alle adolescenti laiche, in Prediche alle donne del secolo XIII. Testi di Umberto a Romans, Gilberto da Tournai, Stefano di Borbone, C. Casagrande (cur.), Milano 1978, p. 20.

25 Cfr. Gen 34.

26 Egidio Romano, Del reggimento de 'principi, F. Corazzini (cur.), Firenze 1858, p. 184.

27 Francesco da Barberino, Reggimento e costumi delle donne, op. cit., pp. 173-174.

28 Gilberto da Tournai, Terza predica alle donne sposate, op.cit., pp. 83-84.

29 C. Casagrande, La donna custodita, in Storia delle donne in Occidente. Il Medioevo, cur. Ch. Klapisch-Zuber, Roma-Bari 2005, p. 106.

30 F. Barberino, Reggimento e costumi di donne, op.cit., p. 53.
} 
di vedere quale modo di ridere la stesse meglio ${ }^{31}$ ingannava l'uomo. Inoltre il predicatore francese vedeva l'inganno anche nella tintura dei capelli $3^{2}$. Con parole forti trattava del trucco delle ragazze insistendo che esse truccandosi il viso insultavano il Creatore ${ }^{33}$. Inoltre per farle riflettere sul loro comportamento faceva loro le seguenti domande: "Se sei bella, perché copri con l'unguento la bellezza del volto? Se sei brutta, perché fingi di essere bella?"34 Dalle sue parole si nota che ogni prova di miglioramento del proprio aspetto da parte della ragazza era, agli occhi di Gilberto, un vero inganno, poiché il potenziale marito non la vedeva così come lei era in realtà, ma solo come un quadro falso.

Riguardo alla bellezza Gilberto sosteneva che la donna non doveva vantarsi del suo corpo e le diceva "se sei bella, non sei nient'altro che un cumulo di sterco coperto di neve" 35 . Invece quando una di loro aveva $\mathrm{i}$ bei capelli, in quel caso doveva sempre ricordarsi che "i capelli sono il capestro diabolico con cui vieni trascinata all'inferno" ${ }^{26}$.

\section{La moglie}

Rivolgendosi alle donne sposate Gilberto di Tournai diceva che esse dovevano essere in grado di governare la casa "che consiste di quattro compiti: nei riguardi dei figli, dei servi, delle ancelle e dei lavori domestici" 37 . Come si nota, l'impegno più importante delle donne sposate che avevano dei figli riguardava proprio la loro educazione. Infatti, il figlio finché era piccolo rimaneva nelle stanze della madre imparando da lei soprattutto le buone maniere e l'educazione religiosa. In seguito passava sotto le ali del padre o di un altro uomo il quale gli insegnava tutto quello che era ritenuto necessario. Sotto la custodia della madre rimanevano invece le figlie, che passavano le loro giornate ad imparare da lei come badare ai fratelli più piccoli, tessere, ricamare, guidare la casa, etc., ossia tutti i doveri che una futura moglie doveva svolgere nella casa del marito. Anche se era ben visto che una donna si occupasse dei lavori domestici, non da tutte le ragazze ci si aspettava la capacità di tessere o filare. Infatti, le discendenti delle famiglie di alto status sociale frequentemente occupavano il loro tempo dedicandolo alla lettura ${ }^{3}$.

Una buona madre doveva ricordare sempre che l'educazione dei figli riguardo alla fede e ai buoni costumi doveva cominciare già dalla tenera età, perché, come sosteneva Gilberto, tutto ciò che il bambino "non imparò nell'infanzia, a fatica si poté apprenderlo in vecchiaia"39. Rivolgendosi alle madri il predicatore francese le istruiva di ricordare ai loro figli "di non ascoltare nient'altro e a non parlare di niente'altro se non di ciò che riguarda il timore

\footnotetext{
31 Cfr. Gilberto da Tournai, Terza predica alle donne sposate, op.cit., p. 84.

32 Cfr. Ibidem, pp. 83-84.

33 Cfr. Ibidem, p. 84.

34 Ibidem.

35 Ibidem.

36 Ibidem.

37 Ibidem, p. 63.

38 Egidio Romano nel Reggimento dice: il tessere, ,...il filare e opere di seta, paiono che sieno opere convenievoli a la femina. E s'elli fuisse alcuna femmina, sì alta o sì nobile, ch' a lei non si convenisse nè tessere nè filare nè opera di seta, o per la dignità di lei o per lo costume del paese, sì le die l'uomo fare leggiare alcun libro o imparare alcuna scienza utile". Egidio Romano, Del reggimento de 'principi, F. Corazzini (cur.), Firenze 1858, p.187.

39 Gilberto da Tournai, Terza predica alle donne sposate, op.cit., p. 64.
} 
e l'amore di Dio"'40. Inoltre esse dovevano influenzare i loro figli a non "avere turpi pensieri" e a "ignorare le canzoni del mondo"42.

Un'altra questione non meno importante riguardava la compagnia della prole. Infatti, una madre aveva il dovere di scegliere con molta cautela quali persone dovessero frequentare i propri figli, perché era necessario diminuire la presenza di quelli che pensavano troppo alle cose mondane: "Allontani le figlie da quelle persone di mondo che possono loro insegnare in modo ancor peggiore il male che hanno acquisito"43. Inoltre una madre aveva il dovere di custodire sua figlia e di ricordarle di conservare la verginità per il marito. Non stupisce allora che Gilberto con ira si rivolgeva alle madri che trascuravano la questione della verginità delle loro figlie o addirittura organizzavano incontri per loro: "Maledette le donne che vendono le loro figlie" 44 .

Inoltre secondo l'insegnamento di Gilberto, una madre doveva ricordarsi del suo dovere di essere un modello da seguire per le figlie. Lo stesso pensiero si può trovare anche nelle poesie di Eustache Deschamps († I407), il quale invitava la figlia a seguire sua madre, perché essa era economa, elegante, dirigeva con cura la casa e i servitori ${ }^{45}$.

Oltre all'educazione dei bambini, una buona moglie doveva dedicare il suo tempo alla guida della casa. Rivolgendosi alle donne che avevano nella casa dei servi, Gilberto le avvertiva dicendo che esse dovevano scacciare di casa i servi giovani e lascivi ${ }^{46}$, perché "i servi malvagi cercano il modo di circuire e raggirare le loro padrone"47. Inoltre Gilberto avvertiva che nelle case si trovavano frequentemente " $i$ servi sfrontati che non hanno modestia nei gesti, né pudore nel vestirsi, né misura nel mangiare, né verecondia nel parlare" ${ }^{28}$. Nel caso trovasse un servo fedele, una donna doveva trattarlo con amicizia e dolcezza ${ }^{49}$. Per rafforzare ancora di più la sua idea al riguardo, il predicatore riportava le parole di san Gerolamo, secondo il quale la donna doveva proteggere i suoi servi come se fosse non la loro padrona ma la loro madre ${ }^{50}$. Non tutti però concordavano con questo pensiero e così ad esempio un autore anonimo del trecento, nel testo intitolato I dodici avvertimenti che deve dare la madre alla figlia quando la manda a marito, nel decimo comandamento avverte la ragazza di non essere "troppo domestica colla tua famiglia, né troppo inchinevole specialmente a quelle persone che ti dovrebbero servire, lo donzello, o servigiale, che sia servo, o serva; perocché troppa dimestichezza importa vizio, e troppa familiarità ingenera sdegno"5I .

Nella casa di una buona moglie doveva sempre regnare il pudore e "una donna saggia deve fare molta attenzione che un servo e un'ancella non macchino la sua casa con la loro

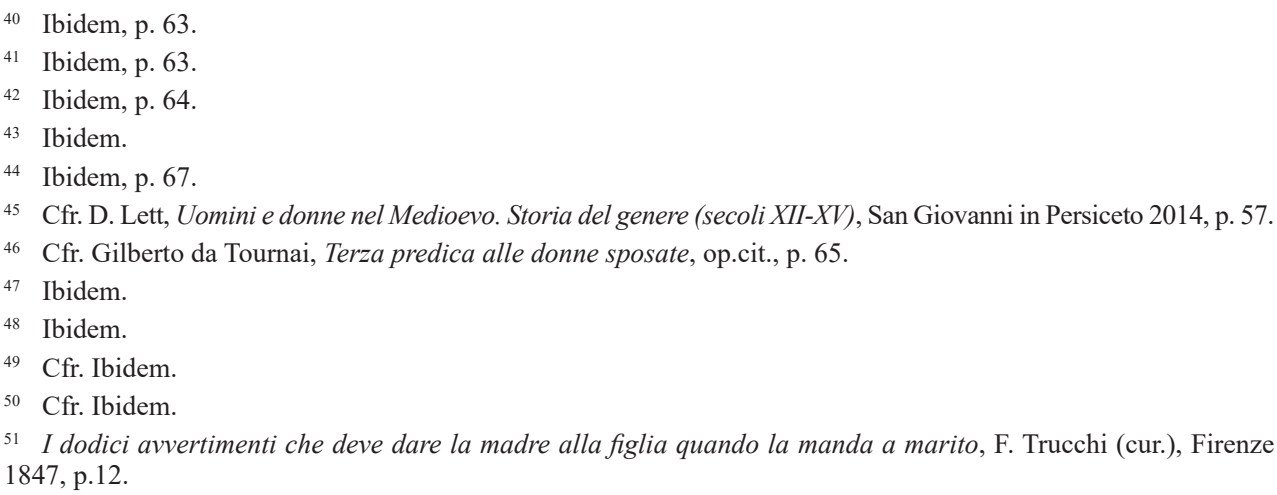


incontinenza" ${ }^{\prime 2}$. La questione della servitù doveva creare molti problemi nelle case poiché Gilberto più di una volta avvertiva la donna di avere "molta attenzione ai servi e alle ancelle perché abitano nella stessa casa, mangiano insieme, si guardano l'un l'altro, hanno i letti vicini"s3. Inoltre dalla donna si esigeva la capacità di rimproverare "le ancelle disordinate e sfrontate" ${ }^{\prime 4}$. Una padrona, secondo Gilberto, doveva essere severa con un'ancella che non le era obbediente, faceva discorsi frivoli o tentava di corrompere sia lei sia le sue figlie ${ }^{55}$. Invero, udendo dalla sua serva le parole "Signora, c'è un cavaliere molto bello, onesto e degno di essere amato, che vi ama" 56 , la padrona aveva il dovere di chiamare le altre serve e frustarla alla loro presenza per far loro capire che lei non tollera questi suggerimenti ${ }^{57}$.

Gilberto nelle sue prediche divideva gli impegni domestici tra la moglie e il marito, spiegando però che mentre quelli della sposa si svolgevano tra le mura domestiche, quelli del marito riguardavano gli affari fuori casa. Invero il marito "deve cercare da mangiare e di che vestirsi mentre la donna dentro le mura domestiche deve occuparsi della cura della casa e dei prodotti degli animali" ${ }^{\prime} 8$. Simili parole si trovano anche nel testo di Dodici avvertimenti dove si legge "che all'uomo bisogna provvedere a' fatti di fuori casa, per fare quelli di dentro alla casa"s9.

L'ultimo, ma non il meno importante dovere della donna, era di "applicarsi alla meditazione delle verità divine" ${ }^{\prime 60}$. Infatti, Gilberto suggeriva alle mogli di possedere nella loro casa una stanza dove potevano passare il loro tempo dedicandolo alla preghiera e al lavoro ${ }^{61}$.

\section{La vedova}

Uno dei principali doveri delle vedove, secondo Gilberto, era quello di condurre una vita santa per diventare un modello da seguire per le altre donne ${ }^{62}$. Inoltre nella società la vedova si doveva conoscere dal "volto pallido" ${ }^{3} 3$, "dell'abito del lutto" ${ }^{64} \mathrm{e}$ dalla buona reputazione ${ }^{65}$.

Le donne rimaste vedove, secondo Gilberto, non potevano abbandonare i loro impegni verso le loro famiglie ${ }^{66}$. Infatti, come prima avevano il compito di educare i figli allora, adesso dovevano fare lo stesso con i figli o i nipoti e se poi non li avevano, allora erano obbligate di ricordarsi dei poveri ${ }^{67}$.

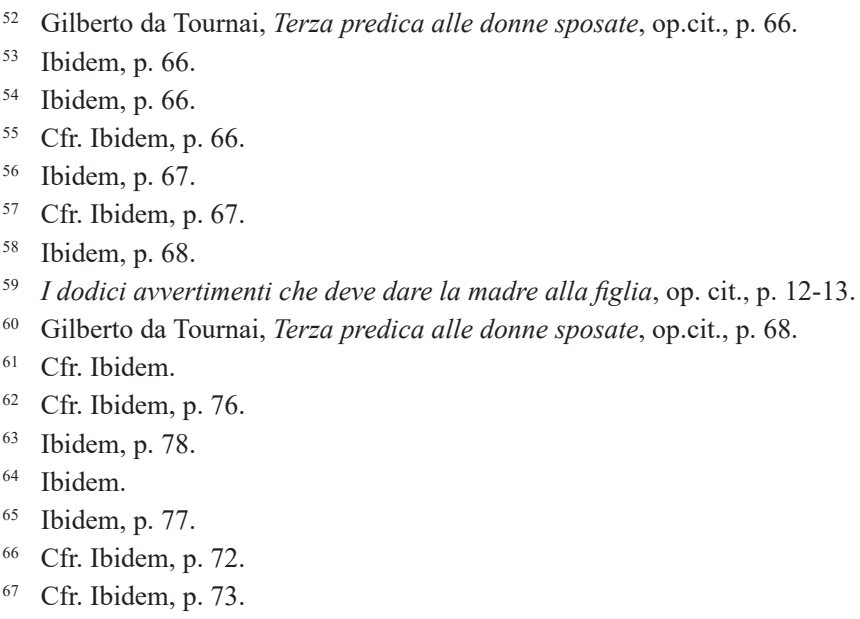


Dalla vedova Gilberto esigeva inoltre una "devozione verso Cristo, pallore per i continui digiuni" ${ }^{6}$, perché chi "avrà i digiuni al posto dei giochi, le preghiere al posto dei piaceri sarà benedetto da Dio" "69. Inoltre il predicatore francese insegnava che a parte la carità, una vedova doveva essere modesta e moderata in tutto quello che faceva ${ }^{70}$ e inoltre la chiesa doveva diventare il suo rifugio più frequentato ${ }^{71}$.

Anche se erano vedove agli occhi degli uomini, rimanevano sempre figlie di Eva, ma si aspettava da loro la vittoria sul desiderio carnale ${ }^{72}$. Esse dovevano rinunciare ai piaceri mondani e dedicare ogni momento della loro vita alla preghiera e al digiuno. Secondo Gilberto nel caso in cui una giovane donna diventasse vedova, si esigeva da lei, tra l'altro, di dimenticare la morbidezza delle piume e d'evitare i bagni caldi perché tutto ciò potrebbe riscaldare "il suo sangue ancora giovane" 73 . Inoltre il predicatore francese non era d'accordo sulle seconde nozze che, nella sua opinione creavano soltanto problemi sia alla donna, sia alla sua precedente famiglia che a quella nuova. Infatti, per essere ancora più convincente riportava le parole di Girolamo: "Avrai dei figli dal secondo marito, porterai la guerra in casa $[. .$.$] il tuo sposo sarà geloso del tuo precedente marito morto e se non ne odierai i figli$ sembrerà che ami ancora il padre"74. Inoltre Gilberto le avvertiva che il posto di seconda moglie era pericoloso, perché avrebbe potuto essere sempre facilmente accusata dalla gente di avvelenare i figli del marito avuti dalla prima moglie quando essi si sentiranno male ${ }^{75}$. Infatti, per evitare tutti questi problemi era meglio, secondo lui, per una vedova rimanere nel suo stato di vedovanza per il resto della sua vita.

\section{Conclusione}

Con il presente studio si intende presentare il concetto di Gilberto di Tournai sui comportamenti e doveri di una ragazza, di una moglie e di una vedova. Grazie alle sue prediche si può vedere quali atteggiamenti delle donne erano, dalla società del tempo, ritenuti giusti e quali invece sbagliati. Grazie alle parole di questo grande predicatore francese si può vedere quali aspettative gli uomini avevano dalle donne per le quali avevano scelto la vita nel mondo e non in convento. Sfortunatamente per mancanza delle testimonianze scritte dalle donne, è difficile dire oggi con certezza come esse reagissero ascoltando le parole dei confessori, predicatori, poeti o scrittori. Si può soltanto supporre che non sempre erano d'accordo con le loro idee, ma educate fin da piccole ad essere sottomesse all'uomo tacevano per paura e per obbedienza. Dovettero passare molti anni prima che una di loro, Cristina da Pizan, avesse il coraggio di affrontare i principali pensatori della sua epoca scrivendo le lettere contro l'opera, così apprezzata dagli uomini, come il Poema della Rosa di Guillaume de Loris e Jean de Meung.

\footnotetext{
68 Ibidem, p. 73.

69 Ibidem, pp. 74-75.

70 Cfr. Ibidem, p. 73.

${ }^{71}$ Cfr. Ibidem.

72 Ibidem, p. 74.

73 Ibidem, p. 78.

74 Ibidem, p. 79.

75 Cfr. Ibidem.
} 


\section{How should the woman behave and what are her duties?}

The guidelines for women presented in the selected sermons of Gilbert of Tournai Summary

The article presents the opinions of the I3th century preacher Gilbert of Tournai about the duties of girls, wives and widows. Gilbert seeing the great lack of women's education addressed his sermons to them and indicated which behaviors were correct and which they should change or avoid. His sermons show how the women was seen, which was her position and role in the $13^{\text {th }}$ century French society.

Keywords: women, Gilbert of Tournai, behavior, obligation, middle age

W jaki sposób powinna zachować się kobieta i jakie są jej obowiązki? Wskazówki dla kobiet zawarte w wybranych kazaniach Gilberta z Tournai Abstrakt

W artykule zostały przedstawione poglądy francuskiego kaznodziei z XIII wieku Gilberta z Tournai na temat obowiązków dziewcząt, żon oraz wdów. Gilbert widząc wielkie braki w kwestii edukacji kobiet zwracał się do nich w swoich kazaniach i wskazywał, jakie zachowania były właściwe, a które należało zmienić albo unikać. Jego kazania przybliżają sposób postrzegania kobiety, jej miejsca i roli w XIII wiecznym społeczeństwie francuskim.

Słowa kluczowe: kobieta, Gilbert z Tournai, zachowanie, obowiązki, średniowiecze

Nota o Autorze: Dr Anna Głusiuk, adiunkt w Katedrze Średniowiecza w Instytucie Nauk Historycznych i Społecznych Uniwersytetu Kardynała Stefana Wyszyńskiego. Zainteresowania naukowe: literatura średniowieczna, sytuacja i rola kobiety w epoce średniowiecza. 